\title{
Rapidly switching multidirectional defibrillation: Reversal of ventricular fibrillation with lower energy shocks
}

\author{
Marcelo A. Viana, MEE, ${ }^{a}$ Rosana A. Bassani, ScD, ${ }^{\mathrm{b}}$ Orlando Petrucci, MD, PhD, ${ }^{\mathrm{c}}$ \\ Denilson A. Marques, BEE, a and José Wilson M. Bassani, $\mathrm{PhD}^{\mathrm{a}, \mathrm{b}}$
}

\begin{abstract}
Objectives: Cardiac arrest after open surgery has an incidence of approximately $3 \%$, of which more than $50 \%$ of the cases are due to ventricular fibrillation. Electrical defibrillation is the most effective therapy for terminating cardiac arrhythmias associated with unstable hemodynamics. The excitation threshold of myocardial microstructures is lower when external electrical fields are applied in the longitudinal direction with respect to the major axis of cells. However, in the heart, cell bundles are disposed in several directions. Improved myocardial excitation and defibrillation have been achieved by applying shocks in multiple directions via intracardiac leads, but the results are controversial when the electrodes are not located within the cardiac chambers. This study was designed to test whether rapidly switching shock delivery in 3 directions could increase the efficiency of direct defibrillation.
\end{abstract}

Methods: A multidirectional defibrillator and paddles bearing 3 electrodes each were developed and used in vivo for the reversal of electrically induced ventricular fibrillation in an anesthetized open-chest swine model. Direct defibrillation was performed by unidirectional and multidirectional shocks applied in an alternating fashion. Survival analysis was used to estimate the relationship between the probability of defibrillation and the shock energy.

Results: Compared with shock delivery in a single direction in the same animal population, the shock energy required for multidirectional defibrillation was $20 \%$ to $30 \%$ lower $(P<.05)$ within a wide range of success probabilities.

Conclusions: Rapidly switching multidirectional shock delivery required lower shock energy for ventricular fibrillation termination and may be a safer alternative for restoring cardiac sinus rhythm. (J Thorac Cardiovasc Surg 2014;148:3213-8)

External, high-intensity electrical fields are applied to the heart for resuscitation from cardiac arrest due to severe disturbances of cardiac rhythm, such as ventricular fibrillation (VF). This approach is used not only in the emergency clinical setting but also during or after cardiac surgery, of which cardiac arrest is the most common complication (incidence of $1 \%-3 \%) .{ }^{1,2}$ Although electrical defibrillation is considered to be the most effective procedure for VF reversal, it has limited efficacy. ${ }^{3}$ Because high-intensity shocks are required for effective defibrillation, consistent with the proposal that arrhythmia reversal requires the excitation of a critical mass of myocardial cells, ${ }^{4,5}$ this procedure may cause a

\footnotetext{
From the Department of Biomedical Engineering, ${ }^{\text {a }}$ School of Electrical and Computer Engineering; Center for Biomedical Engineering ${ }^{\mathrm{b}}$; and Department of Surgery, ${ }^{\mathrm{c}}$ Faculty of Medical Sciences, University of Campinas (UNICAMP), Campinas, São Paulo, Brazil.

J.W.M.B. received a grant (300632/2005-3) and M.A.V. received a scholarship (135204/2008-9) from the Conselho Nacional de Pesquisa e Desenvolvimento, (CNPq), Brasilia, DF, Brazil.

Disclosures: Authors have nothing to disclose with regard to commercial support. Received for publication April 14, 2014; revisions received June 25, 2014; accepted for publication July 7, 2014; available ahead of print Aug 28, 2014

Address for reprints: Orlando Petrucci, MD, PhD, Faculty of Medical Sciences, Department of Surgery, UNICAMP, R. Tessália Vieira de Camargo, 126, 13083-887 Campinas, São Paulo, Brazil (E-mail: petrucci@unicamp.br). $0022-5223 / \$ 36.00$

Copyright (C) 2014 by The American Association for Thoracic Surgery http://dx.doi.org/10.1016/j.jtcvs.2014.07.035
}

variable degree of myocardial damage, mostly due to electroporation of the cardiomyocyte sarcolemma. Such damage, which can be produced by electrical fields even considerably weaker than those reached during electrical defibrillation, ${ }^{6,7}$ may result in transient diastolic depolarization, reduction of the action potential amplitude, depressed ejection, conduction block, $\mathrm{Ca}^{2+}$ overload, and cell injury. ${ }^{6-9}$ The intensity of the peak electrical current applied was recently identified as the major factor related to the deleterious effects of electrical shocks. ${ }^{10}$ Thus, approaches that can decrease the effective shock intensity would represent an important improvement of this therapy, because they should result in less myocardial damage.

A possible approach to achieve more effective defibrillation is the application of shocks in different directions. This approach may increase the probability that the orientation of the stimulating electrical field is parallel to the major axis of the myocytes or muscle bundle, thus minimizing the stimulus threshold amplitude. ${ }^{9,11-14}$ The use of multiple stimulation directions was shown to optimize excitation in randomly oriented isolated cardiomyocytes. ${ }^{14}$ Moreover, it has been reported that shock delivery in different directions via intraventricular leads increases the efficiency of internal defibrillation. ${ }^{15-18}$ However, it is not yet clear whether this stimulation modality is also more efficient 


\section{Abbreviations and Acronyms \\ UNICAMP $=$ University of Campinas \\ $\mathrm{VF} \quad=$ ventricular fibrillation}

when shocks are applied externally to the heart, which is the case during perioperative cardiac arrest and out-ofhospital emergency defibrillation. Although some authors observed decreases in the energy and peak voltage required to terminate $\mathrm{VF}$ by pulse delivery over more than 1 pathway, ${ }^{16,19,20}$ others did not find improvements compared with a single pathway. ${ }^{21}$

This study was designed to test the hypothesis that rapidly switching shock delivery in 3 directions improves the success of direct defibrillation. To enhance the reproducibility of the stimulus delivery pathways and to simulate the condition of defibrillation during intraoperative cardiac arrest, shocks were applied on the epicardial surface of open-chest swine via 3 electrode pairs located in paddles that encircle the heart.

\section{METHODS}

\section{Instrumentation}

Rapidly switching multidirectional defibrillator. To perform a proper comparison between defibrillation modalities, it is essential that the stimulus waveform be identical in the intensity (voltage and current) and time course for unidirectional and multidirectional stimulation. For this purpose, we developed a defibrillator with a rapidly switching circuit that allows monophasic truncated exponential voltage pulses to be delivered from 3 different pairs of electrodes. ${ }^{14}$ Shock energy ranged from 0.5 to $7.3 \mathrm{~J}$ (equivalent to a discharge voltage from 141 to 382 $\mathrm{V}$, assuming $50 \Omega$ cardiac impedance). A capacitance of $100 \mu \mathrm{F}$ was chosen to minimize the charging voltage and current levels. Figure 1 shows the pulses delivered in the unidirectional and multidirectional stimulation modalities. In the latter case, pulses were sequential, without interval or temporal overlap. Bench tests performed before the in vivo experiments showed that, for a given energy level, the instrument delivered pulses of the same amplitude and time course through 1 or 3 pairs of electrodes within the whole energy range for a resistive load up to $560 \Omega$.

In the present experiments, the duration of the defibrillatory pulse was $20 \mathrm{~ms}$. The rapid switching allowed the delivery of 3 pulses within 60 $\mathrm{ms}$, which is a shorter period than the action potential duration of the swine ventricle at physiologic temperature. ${ }^{22}$ Thus, it is expected that each recruited cell should be excited by only 1 of the shocks. When the unidirectional modality is chosen, trigger pulses were delivered via only 1 output connected to a single (central) pair of electrodes.

Defibrillation handles and paddles. Each handle is composed of a hollow, cylindrical shaft with a nylon grip on the superior extremity. The inferior extremity is connected to a semicircular paddle, transversal to the shaft, to which the electrodes are fixed (Figure 2, A). The paddles were designed to encircle the heart, allowing direct contact between the electrode surface and the ventricular epicardium (Figure 2, B). The handles and paddles were made of polycarbonate/acrylnitrile-butadiene-styrene by rapid prototyping at the Institute of Biofabrication of the Faculty of Chemical Engineering of the University of Campinas (UNICAMP).

The electrodes are concave disks of polished surgical stainless steel (25-mm radius, 1-mm thickness, 2-mm maximal depth in the present version). In addition to an electrode located at the center of each paddle, 2 electrodes are disposed at $60^{\circ}$ from the central electrode to allow the delivery of shocks in 3 directions $\left(0^{\circ}, 60^{\circ}\right.$, and $\left.120^{\circ}\right)$ (Figure 2). Labeled pushbuttons on the top of the handle grips enable the capacitors to be charged or the discharge to be triggered.

\section{In Vivo Defibrillation Experiments}

Experiments were conducted at the Laboratory of Surgical Techniques of the Nucleus of Medicine and Experimental Surgery, Faculty of Medical Sciences of UNICAMP.

Animal preparation. The animal care and experimental protocols were in agreement with the Guide for the Care and Use of Laboratory Animals, consistent with the principles of the Brazilian Society of Laboratory Animal Science, and approved by the Institutional Committee of Ethics in Animal Use, Institute of Biology, University of Campinas (protocol number 2251-1).

Ten female Landrace Large White pigs (aged 8 weeks, $20 \pm 3 \mathrm{~kg}$ ) were maintained in individual bays at the animal facility of the Nucleus of Medicine and Experimental Surgery, Faculty of Medical Sciences of UNICAMP, where they were offered filtered water and industrial chow ad libitum. Animals were not subjected to any kind of experimental procedure or restriction (eg, fasting) before the experiment.

After sedation with intramuscular ketamine $\left(10 \mathrm{mg} \cdot \mathrm{kg}^{-1}\right)$, animals were anesthetized with fentanyl hydrochloride $\left(12.5 \mu \mathrm{g} \cdot \mathrm{kg}^{-1}\right)$ and sodium thiopental $\left(25 \mathrm{mg} \cdot \mathrm{kg}^{-1}\right)$, administered as a bolus via the ear vein. After orotracheal intubation, the animals were artificially ventilated $\left(10 \mathrm{ml} \cdot \mathrm{min}^{-1} \cdot \mathrm{kg}^{-1}\right.$ body weight, $\left.50 \% \mathrm{O}_{2}\right)$, and electrodes were attached to the limbs for electrocardiographic monitoring. ${ }^{23}$ After skin disinfection, thoracotomy was performed, followed by pericardium opening and exposure of the heart, which was periodically moistened with saline solution. Anesthesia depth was monitored throughout the experiment, and anesthetic supplementation was performed when necessary. Fluid reposition was performed by Ringer solution infusion via the ear vein $\left(15 \mathrm{~mL} \cdot \mathrm{kg}^{-1} \cdot \mathrm{h}^{-1}\right)$. Fibrillation/defibrillation protocol. Both defibrillation modalities (unidirectional and multidirectional) were tested in each animal by using the up-and-down protocol (described later) in an alternate fashion. This approach was used to minimize the influence of interindividual differences and other factors that might affect the sensitivity to VF induction and/or reversal during the course of the experiment.

VF was induced by brief low-energy DC stimulation of the left ventricle epicardial surface. ${ }^{24}$ Immediately before VF induction, the paddles were positioned in a basoapical orientation for full contact of the electrodes with the ventricular surface (Figure 2, B). Approximately 15 seconds after electrocardiographic confirmation of VF establishment and interruption of coordinated ventricular contractions, a defibrillatory shock of one of the modalities was applied. If it successfully restored the sinus rhythm, then VF was reinduced later, and defibrillation was attempted with a shock of the same modality with lower energy. Otherwise, VF was terminated by stimulation with a conventional defibrillator (Cardiomax, Instramed, Porto Alegre, RS, Brazil), and a shock of the same modality and higher energy was used in the next trial. Afterward, the protocol was repeated for the other defibrillation modality. Between trials, a recovery period was observed (typically 5-15 minutes) until the heart rate was stable and close to its basal value. The goal of this procedure was to obtain pairs of shock energy values (ie, effective and ineffective at reverting VF) for each modality.

The modalities were alternated until the animals developed persistent tachycardia, spontaneous VF initiation, and/or refractoriness to defibrillation. In any of these cases, anesthesia was deepened with thiopental, and the animal was euthanized by intracardiac injection of $3 \mathrm{~mol} / \mathrm{L} \mathrm{KCl}$ solution. Experiments lasted 2 to 4 hours.

\section{Data Analysis}

Data are presented as the mean and standard error of the mean. Pairs of shock energy values were used as the primary data for the survival analysis, which was performed with both pooled data and individual data from some animals. The resulting relationships between the probability of successful 

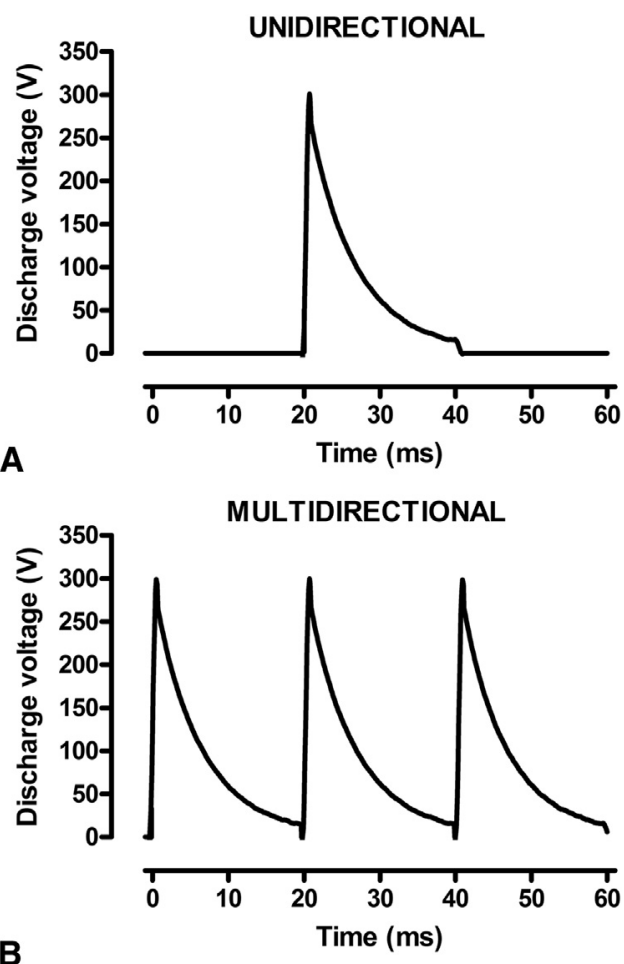

FIGURE 1. Five $J$ discharge pulses of the defibrillator in the unidirectional (A) and multidirectional (B) modes.

defibrillation and the shock energy for the unidirectional and multidirectional modalities were compared with the Mantel-Cox test. A sigmoidal function was fitted to this relationship $\left(\mathrm{R}^{2}>0.98\right)$, and the curve parameters were compared by the F-test. From this function, it was possible to estimate the shock energy values required for different probabilities of successful defibrillation in individual animals, which were compared between modalities with the Student $t$ test for paired samples. Statistical significance was considered for $P<.05$.

\section{RESULTS}

The average heart rates before VF induction and after post-defibrillation stabilization were $100 \pm 3$ beats/min and $101 \pm 3$ beats/min, respectively. The cardiac impedance, estimated from the time constant of the voltage decay during a defibrillatory pulse $(6.92 \pm 1.09 \mathrm{~ms})$, was $61 \pm 11 \Omega$. This value is not significantly different
( $P>.35$, Dunnett's test) from the value of $50 \Omega$ that was assumed in the project of the defibrillator.

Figure 3, A shows the defibrillation curves (pooled data) for unidirectional and multidirectional shocks. The curve parameters are presented in Table 1. Compared with the curve obtained with unidirectional shocks, the curve for multidirectional defibrillation showed a parallel $(P>.05$ for Hill coefficient, F-test) leftward shift. A significant difference was observed between the curves for the 2 defibrillation modalities $(P<.003$; Mantel-Cox test). The shock energy required for successful defibrillation in $50 \%$ of cases $\left(\mathrm{E}_{50}\right.$ value) was approximately $30 \%$ lower for multidirectional stimulation $(P<.001$, F-test) (Table 1 and Figure 3, A). These results indicate that, for shocks of a given energy level, the latter modality was associated with a higher probability of defibrillation than the conventional, unidirectional stimulation.

In some cases, it was possible to obtain from a single animal a sufficient number of data points to determine defibrillation curves for both stimulation modalities. In these instances, it was possible to perform a statistical comparison of the energy level required for defibrillation probabilities greater than .5 , which are clinically more relevant. Despite a large variability among the animals, it was observed that, even for $90 \%$ defibrillation success, the required energy was at least $20 \%$ lower for multidirectional compared with unidirectional shocks $(P<.05$, paired $t$ test; $\mathrm{N}=6$ ) (Figure 3, $B$ ).

\section{DISCUSSION}

We used an in vivo porcine model that is widely used for studies of cardiovascular diseases and therapies. ${ }^{25}$ The results showed that delivery of shocks in 3 directions in rapid succession significantly decreased the stimulus intensity required for successful direct defibrillation compared with shock delivery in a single direction. Thus, our initial hypothesis was confirmed.

One rationale for multidirectional stimulation is based on the spatial interaction of electrical fields and myocardial microstructures. The excitation threshold for field application transversal to the major axis of a cell or cardiomyocyte

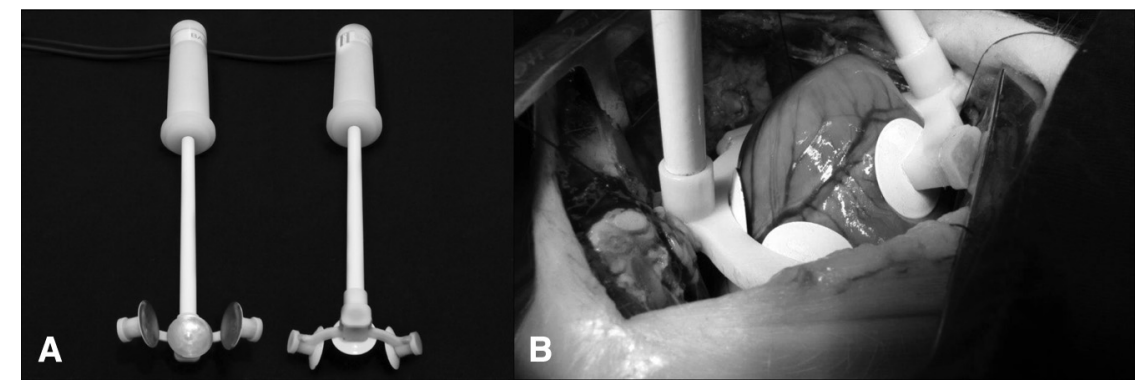

FIGURE 2. Handles/paddles designed for multidirectional defibrillation (A). Each paddle contains 3 electrodes. When the electrodes encircle the heart (B), shocks may be delivered in 1 or 3 directions. 


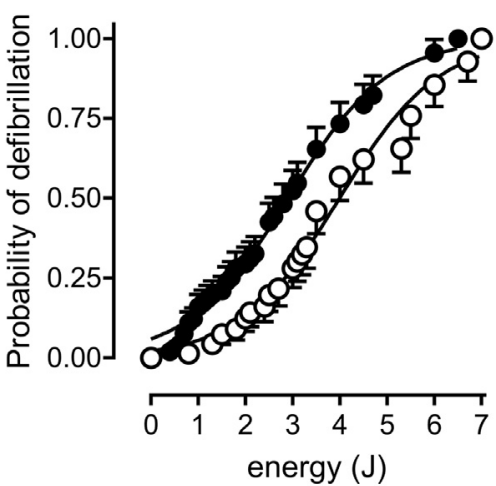

A

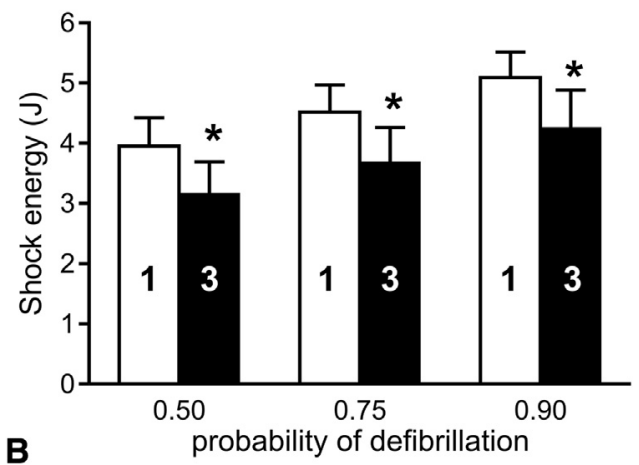

FIGURE 3. A, Defibrillation curves determined in vivo in swine by direct application of unidirectional and multidirectional shocks. Points are mean \pm standard error of the mean values obtained from the survival analysis. Curve parameters are shown in Table 1. Mantel-Cox test indicated significant difference between the curves $(P<.003)$. B, Shock energy levels associated with defibrillation success in $50 \%, 75 \%$, and $90 \%$ of cases for both defibrillation modalities (number of directions of shock delivery is indicated in the bars) determined in a single set of animals $(\mathrm{N}=6)$. Data are shown as the mean and standard error of the mean. ${ }^{*} P<.05$, Student $t$ test for paired samples.

bundle may be 2-fold greater than that for longitudinal field application. ${ }^{9,11-14}$ According to theoretic and experimental studies, this direction-dependent sensitivity to electrical fields stems from the ability of the latter to produce membrane polarization: A lower stimulus intensity is required for a given change in membrane potential (eg, threshold depolarization) in the longitudinal than in the transversal field orientation. ${ }^{9,13,26}$ Because the fiber bundles and sheets of the ventricular walls and septum are

TABLE 1. Defibrillation curve parameters determined in vivo in swine using direct unidirectional and multidirectional defibrillatory shocks

\begin{tabular}{lccc}
\hline $\begin{array}{c}\text { Defibrillation } \\
\text { modality }\end{array}$ & $\begin{array}{c}\text { Hill coefficient } \\
\left(\mathbf{J} \cdot \mathbf{1 0}^{-\mathbf{1}}\right)\end{array}$ & $\mathbf{E}_{\mathbf{5 0}} \mathbf{( J )}$ & $\begin{array}{c}\text { Degrees of } \\
\text { freedom }\end{array}$ \\
\hline Unidirectional & $0.401 \pm 0.021$ & $4.01 \pm 0.06$ & 20 \\
Multidirectional & $0.424 \pm 0.014$ & $2.89 \pm 0.03^{*}$ & 27 \\
\hline
\end{tabular}

Data are presented as mean \pm standard error of the mean. $E_{50}$ : shock energy required for successful defibrillation in $50 \%$ of cases. ${ }^{*} P<.05$ for unidirectional versus multidirectional defibrillation (F-test). highly branched and disposed in several spatial orientations, ${ }^{27}$ stimulation in a single direction should be capable of electrically recruiting only a limited number of cells, unless the stimulus intensity is considerably higher, as was previously observed in vitro. ${ }^{14}$ Therefore, stimulation in different orientations should increase cell recruitment and, thus, the probability of extinguishing reentrant pathways. In addition, it might decrease the probability of post-shock reentrant waves because of the successive establishment of virtual electrodes with different polarities. $^{28}$

Although some authors have shown the superiority of shock delivery through 2 pathways over unidirectional defibrillation, most studies have dealt with stimulation via intracardiac leads. ${ }^{15,16,18,19}$ For shock delivery via epicardial or transthoracic pads, studies have reported a decrease $^{16,19,20}$ or no change ${ }^{21}$ in the shock energy requirement for defibrillation for pulse application over more than 1 pathway. Moreover, the use of 3 directions in a single defibrillation event has not been explored much, except by Pagan-Carlo and colleagues, ${ }^{20}$ who reported facilitation of transthoracic defibrillation in swine and dogs by the use of 6 versus 2 electrode pads. Our present results with direct cardiac stimulation are consistent with the latter findings, although the temporal shock presentation was different (discussed later).

An important aspect when considering multidirectional stimulation is the timing of shock delivery. In all studies that have demonstrated greater defibrillation effectiveness for sequential shocks in more than 1 direction, the interval between shocks was short $\left(\leq 1 \mathrm{~ms}^{15-18}\right)$ or null (present experiments), whereas no improvement was observed for long coupling intervals $\left(100 \mathrm{~ms}^{21}\right)$. This result might be due to the interaction of 2 independent factors: (1) the optimization of the alignment of the electrical field with the major axis of the cell and (2) the possible summation of subthreshold membrane depolarization responses of cells that are only partially aligned to the field, as previously observed in isolated ventricular myocytes. ${ }^{14}$

Some authors have reported an even greater decrease in the defibrillation energy requirement when the pulses applied in different pathways overlap in time. ${ }^{19,20}$ However, although the increase in electrical current flowing during the overlap period may further favor excitation, it also may increase the risk of injury to cells aligned with the electrical vector created by the overlapping stimuli. Cells stimulated with longitudinal fields are more sensitive not only to excitation but also to lethal injury by external fields. ${ }^{9}$ Moreover, the peak current level, rather than the shock energy, seems to be related to the production of deleterious effects in cardiomyocytes. ${ }^{10}$ In our case, the total delivered energy was greater with the multidirectional approach. However, because the temporal shock presentation precluded vectorial summation of the 
applied electrical field, and the waveforms were identical for unidirectional and multidirectional shocks of the same energy level, the reduction of the shock energy requirement in the latter modality translated into a reduction of the peak current applied during a shock.

\section{Study Limitations}

Electrical defibrillation was performed under general anesthesia, after a short VF period, in the absence of cardiovascular disease or myocardial ischemia. However, this model mimics the scenario of electrical defibrillation during cardiac surgery. This aspect is especially true in terms of the use of direct defibrillation, which allowed standardization of electrode positioning and, thus, greater reliability of the results. On the other hand, the possible effects of the anesthesia on cardiovascular and respiratory functions should not have biased the results, because both defibrillation modalities were tested in the same animals. It remains to be determined whether the difference between the defibrillation modalities is maintained after long-lasting VF. In addition, we used monophasic defibrillation shocks, whereas the most recent guidelines on resuscitation recommend biphasic shocks because of their reportedly greater efficiency, ${ }^{29}$ although some studies have failed to find a significant influence of the waveform on the outcome of patients. ${ }^{30,31}$ Monophasic stimuli were chosen in this study because they allow determining the actual effect of multidirectional stimulation in the absence of possible confounding effects associated with the biphasic waveform, which may be clarified in future studies. Moreover, monophasic defibrillatory shocks are still frequently used in clinical practice. ${ }^{30,31}$

\section{CONCLUSIONS}

The present results with epicardial shock delivery indicate that rapidly switching, multidirectional defibrillation is more effective than unidirectional, conventional defibrillation, because it requires lower shock energy levels for successful restoration of the sinus rhythm. For direct defibrillation, which is the case in open chest surgeries, the 3-electrode paddle system is easy to use, and, given the requirement of lower shock energy for successful VF reversal, multidirectional defibrillation may represent a promising alternative for the safer electrical therapy of cardiac arrhythmias.

The authors thank Mauro Martinazo, Renato S. Moura, BE, and Carlos A. Silva, BTech (Center for Biomedical Engineering, UNICAMP), Ana Cristina de Moraes, and William A. Silva (Faculty of Medical Sciences, UNICAMP), for excellent technical support during the development and testing of the defibrillator; and the Institute of Biofabrication of the Faculty of Chemical Engineering of UNICAMP for the contribution to the development of the paddles.

\section{References}

1. Dunning J, Fabbri A, Kolh PH, Levine A, Lockowandt U, Mackay J, et al. Guideline for resuscitation in cardiac arrest after cardiac surgery. Eur $J$ Cardiothorac Surg. 2009;36:3-28.

2. Vanden Hoek TL, Morrison LJ, Shuster M, Donnino M, Sinz E, Lavonas EJ, et al. Part 12: cardiac arrest in special situations: 2010 American Heart Association Guidelines for Cardiopulmonary Resuscitation and Emergency Cardiovascular Care. Circulation. 2010;122:S829-61.

3. Walker RG, Koster RW, Sun C, Moffat G, Barger J, Dodson PP, et al. Defibrillation probability and impedance change between shocks during resuscitation from out-of-hospital cardiac arrest. Resuscitation. 2009;80:773-7.

4. Zipes DP, Fischer J, King RM, Nicoll Ad, Jolly WW. Termination of ventricular fibrillation in dogs by depolarizing a critical amount of myocardium. Am J Cardiol. 1975;36:37-44.

5. Chapman PD, Sagar KB, Wetherbee JN, Troup PJ. Relationship of left ventricular mass to defibrillation threshold for the implantable defibrillator: a combined clinical and animal study. Am Heart J. 1987;114:274-8.

6. Yabe S, Smith WM, Daubert JP, Wolf PD, Rollins DL, Ideker RE. Conduction disturbances caused by high current density electric fields. Circ Res. 1990;66: 1190-203.

7. Fedorov VV, Nikolski VP, Efimov IR. Effect of electroporation on cardiac electrophysiology. Methods Mol Biol. 2008;423:433-48.

8. Hendrikx M, Jiang H, Gutermann H, Toelsie J, Renard D, Briers A, et al. Release of cardiac troponin I in antegrade crystalloid versus cold blood cardioplegia. J Thorac Cardiovasc Surg. 1999;118:452-9.

9. de Oliveira PX, Bassani RA, Bassani JW. Lethal effect of electric fields on isolated ventricular myocytes. IEEE Trans Biomed Eng. 2008;55:2635-42.

10. Tsai MS, Tang W, Sun S, Wang H, Freeman G, Chen WJ, et al. Individual effect of components of defibrillation waveform on the contractile function and intracellular calcium dynamics of cardiomyocytes. Crit Care Med. 2009; 37:2394-401.

11. Tung L, Sliz N, Mulligan MR. Influence of electrical axis of stimulation on excitation of cardiac muscle cells. Circ Res. 1991;69:722-30.

12. Knisley SB, Baynham TC. Line stimulation parallel to myofibers enhances regional uniformity of transmembrane voltage changes in rabbit hearts. Circ Res. 1997;81:229-41.

13. Bassani RA, Lima KA, Gomes PA, Oliveira PX, Bassani JW. Combining stimulus direction and waveform for optimization of threshold stimulation of isolated ventricular myocytes. Physiol Meas. 2006;27:851-63.

14. Fonseca AV, Bassani RA, Oliveira PX, Bassani JW. Greater cardiac cell excitation efficiency with rapidly switching multidirectional electrical stimulation. IEEE Trans Biomed Eng. 2013;60:28-34.

15. Bourland JD, Tacker WA Jr, Wessale JL, Kallok MJ, Graf JE, Geddes LA Sequential pulse defibrillation for implantable defibrillators. Med Instrum. 1986;20:138-42.

16. Chang MS, Inoue H, Kallok MJ, Zipes DP. Double and triple sequential shocks reduce ventricular defibrillation threshold in dogs with and without myocardial infarction. J Am Coll Cardiol. 1986;8:1393-405.

17. Jones DL, Klein GJ, Guiraudon GM, Sharma AD, Kallok MJ, Bourland JD, et al Internal cardiac defibrillation in man: pronounced improvement with sequential pulse delivery to two different lead orientations. Circulation. 1986; 73:484-91.

18. Exner D, Yee R, Jones DL, Klein GJ, Mehra R. Combination biphasic waveform plus sequential pulse defibrillation improves defibrillation efficacy of a nonthoracotomy lead system. J Am Coll Cardiol. 1994;23:317-22.

19. Kerber RE, Spencer KT, Kallok MJ, Birkett C, Smith R, Yoerger D, et al. Overlapping sequential pulses. A new waveform for transthoracic defibrillation. Circulation. 1994;89:2369-79.

20. Pagan-Carlo LA, Allan JJ, Spencer KT, Birkett CL, Myers R, Kerber RE Encircling overlapping multipulse shock waveforms for transthoracic defibrillation. J Am Coll Cardiol. 1998;32:2065-71.

21. Kerber RE, Bourland JD, Kallok MJ, Hite P, Pritchard B, Charbonnier F, et al. Transthoracic defibrillation using sequential and simultaneous dual shock pathways: experimental studies. Pacing Clin Electrophysiol. 1990;13: 207-17.

22. Roscher R, Arlock P, Sjoberg T, Steen S. Effects of dopamine on porcine myocardial action potentials and contractions at 37 degrees $\mathrm{C}$ and 32 degrees C. Acta Anaesthesiol Scand. 2001;45:421-6.

23. Petrucci Junior O, Oliveira PP, Carmo MR, Vieira RW, Braile DM Standardization of an isolated pig heart preparation with parabiotic circulation: methodological considerations. Braz J Med Biol Res. 2003;36:649-59. 
24. Euler DE, Whitman TA, Roberts PR, Kallok MJ. Low voltage direct current delivered through unipolar transvenous leads: an alternate method for the induction of ventricular fibrillation. Pacing Clin Electrophysiol. 1999;22:908-14.

25. Suzuki Y, Yeung AC, Ikeno F. The representative porcine model for human cardiovascular disease. J Biomed Biotechnol. 2011;2011:195483.

26. Neunlist M, Tung L. Spatial distribution of cardiac transmembrane potentials around an extracellular electrode: dependence on fiber orientation. Biophys J. 1995;68:2310-22.

27. Gilbert SH, Benoist D, Benson AP, White E, Tanner SF, Holden AV, et al. Visualization and quantification of whole rat heart laminar structure using high-spatial resolution contrast-enhanced MRI. Am J Physiol Heart Circ Physiol. 2012;302:H287-98.

28. Efimov I, Ripplinger CM. Virtual electrode hypothesis of defibrillation. Heart Rhythm. 2006;3:1100-2.
29. Koster RW, Baubin MA, Bossaert LL, Caballero A, Cassan P, Castren M, et al. European Resuscitation Council Guidelines for Resuscitation 2010 Section 2. Adult basic life support and use of automated external defibrillators. Resuscitation. 2010;81:1277-92.

30. Shelton RJ, Brown BD, Allinson A, Johnson T, Smales C, Jolly S, et al. A comparison between monophasic and biphasic defibrillation for the cardioversion of persistent atrial fibrillation in patients with and without heart failure. Int J Cardiol. 2011;147:405-8.

31. Tanabe S, Yasunaga H, Ogawa T, Koike S, Akahane M, Horiguchi H, et al. Comparison of outcomes after use of biphasic or monophasic defibrillators among out-of-hospital cardiac arrest patients: a nationwide population-based observational study. Circ Cardiovasc Qual Outcomes. 2012;5:689-96. 